\title{
O limítrofe do horário de verão: análises quase-experimentais do consumo de energia elétrica na Bahia e no Tocantins
}

The daylight saving borderline: quasi-experimental analysis of the electric power consumption in Bahia and Tocantins

\begin{abstract}
The daylight saving time is adopted in Brazil to improve the energy efficiency and the interconnected electrical system safety. Southern states tend to contribute more in this direction, with a questionable boundary in Bahia and Tocantins. Exploring the causal inference model of Neyman-Rubin and some quasi-experimental situations in Bahia and Tocantins, the article analyzes the trajectories of the total state consumption of electric energy using differences-in-differences and synthetic control. It is concluded that the participation of Bahia generates an energy economy of up to $252 \mathrm{GWh}$, and that the participation of Tocantins does not generate significant effects.
\end{abstract}

\section{Keywords}

daylight saving time; total electrical energy consumption; quasi-experiment; differences-in-differences; synthetic control.

JEL Codes C15, E27, Q47.

\author{
Francis Petterini (1) \\ Diogo Signor (2) \\ Pedro Santos ${ }^{(3)}$ \\ (1) Universidade Federal de Santa Catarina \\ (2) Universidade Federal de Santa Catarina \\ (3) Universidade Federal de Santa Catarina
}

\section{Resumo}

O horário de verão é adotado no Brasil para melhorar a eficiência energética e a segurança do sistema elétrico interligado. Os estados meridionais tendem a contribuir mais nesse sentido, havendo um limitrofe questionável em estados como Bahia e Tocantins. Explorando o arcabouço de inferência causal de Neyman-Rubin e situações quase-experimentais na Bahia e no Tocantins, o artigo analisa as trajetórias do consumo total de energia elétrica estadual usando diferenças-em-diferenças e controle sintético. Conclui-se que a participação da Bahia gera uma economia energética de até 252 GWh, e que a participação do Tocantins não gera efeitos significativos.

\section{Palavras-chave}

horário de verão; consumo total de energia elétrica; quase-experimento; diferenças-em-diferenças; controle sintético.

Códigos JEL C15, E27, Q47. 


\section{Introdução}

A ideia básica do horário de verão é aproveitar a iluminação natural dos dias mais longos para economizar energia. Além disso, muitos países adotam a prática para aumentar a segurança do sistema elétrico interligado, pois o atraso do início da iluminação noturna provoca a dispersão dos picos de demanda, evitando sobrecargas. De uma forma geral, o objetivo é usar menos energia per capita para um mesmo nível de atividade econômica (ARIES e NEWSHAM, 2008).

No Brasil a prática foi adotada esporadicamente desde o verão de 1931/32, e passou a ser adotada periodicamente desde o verão 1985/86. Deste verão até o de 1987/88 todas das unidades da federação (UFs) alteram o horário, mas depois do verão de 1988/89 a prática foi experimentada sequencialmente em subconjuntos das UFs ${ }^{1}$. Como os dias de verão são menores nos estados setentrionais em relação aos meridionais, o horário foi experimentado poucas vezes nos primeiros ${ }^{2}$. Assim, considerando a iluminação relativa do sol e a polêmica que o horário de verão gera, já que muda forçosamente a rotina das pessoas, as análises do Operador Nacional do Sistema Elétrico (ONS) indicam que a sua adoção não se justificaria mais ao norte, e há um limítrofe na Bahia, Tocantins e Mato Grosso.

É natural que divergências quanto à adoção do horário diferenciado ocorram nos estados limítrofes, pois a variação da incidência de luz solar não é tão significativa como nos estados mais ao sul, mas é mais que nos demais estados ao norte. Por exemplo, desde o verão de 1988/89, o horário não foi alterado no Mato Grosso uma única vez, entretanto mais de 30 de seus municípios se recusaram recorrentemente a adotá-lo ${ }^{4}$. Por outro lado, o horário foi alterado na Bahia e no Tocantins em 16 e 10 desses últimos verões ${ }^{5}$, respectivamente, sendo que as notícias veiculadas na mídia

1 Na seção 3, a Figura 1 ilustra quantas vezes cada UF experimentou o horário entre os verões de 1988/89 e 2015/16.

2 No primeiro dia do verão, por exemplo, Porto Alegre (a capital mais meridional) é iluminada pelo sol por cerca de duas horas e seis minutos a mais que Boa Vista (a capital mais setentrional).

3 Ver a sequência de relatórios do ONS em www.ons.org.br/avaliacao_condicao/horario_ verao.aspx.

4 Isso pode ser verificado, por exemplo, em uma pesquisa na Internet usando as palavras "Barra das Garças horário de verão" - sendo que Barra das Garças foi o primeiro município a se rebelar contra a mudança do horário.

5 Detalhes sobre quais verões o horário foi adotado em cada UF podem ser consultados no 
sugerem que suas participações têm envolvido razões alheias à eficiência energética. Em particular, recentemente esses dois estados foram quase-experimentados no seguinte sentido:

- Na Bahia o horário foi alterado no verão de 2011/12, mas não nas edições imediatamente anteriores e posteriores, devido a polêmicas entre entidades de classe sobre as vantagens e desvantagens da medida, que pressionaram pró e contra sua adoção $;$ e,

- No Tocantins o horário foi alterado no verão de 2012/13, mas não nas edições imediatamente anteriores e posteriores, com a "intenção de colocar momentaneamente o estado em conformidade com o horário de Brasília no que se refere ao funcionamento do sistema bancário nacional e das tabelas de voos originários do aeroporto de Palmas";

Nesse contexto, levando em conta os quase-experimentos que ocorreram nos casos da Bahia e do Tocantins, este artigo busca contribuir com a análise do limítrofe do horário de verão no Brasil. $\bigcirc$ estudo se apresenta relevante uma vez que há indicativos de efeito causal da prática na economia de energia e água nos reservatórios hidroelétricos, e na redução da probabilidade de "apagões", mas não há uma fronteira de adoção bem estabelecida. Além disso, o artigo também busca contribuir com uma análise alternativa ao ONS, já que aqui se apresenta uma aplicação inédita da abordagem de Neyman-Rubin aos dados brasileiros.

Nessa abordagem o efeito causal é a diferença dos resultados factual e contrafactual de um indicador de impacto. Como é impossível observar ambos concomitantemente, a estratégia é estimar um contrafactual através da comparação de grupos de UFs. Porém, tal comparação identificará o efeito causal somente se esses grupos são determinados por processos experimentais ou similares, por isso se explora os quase-experimentos descritos acima. Dessa forma, usando uma base de dados da Empresa de Pesquisa Energética (EPE), que compila mensalmente e de forma padronizada o consumo agregado de energia elétrica por UFs, com diferenças-em-diferenças e controles sintéticos verificam-se indicativos de que a Bahia deveria adotar o horário de verão, mas o Tocantins não.

levantamento do Observatório Nacional em http://pcdsh01.on.br/DecHV.html.

6 Isso pode ser verificado, por exemplo, em uma pesquisa na Internet usando as palavras "Bahia horário de verão". Embora não se tenha encontrado nenhuma comunicação oficial de órgão governamental sobre o assunto.

7 Ver a comunicação oficial em http://secom.to.gov.br/noticia/87569. 
Além dessa introdução, o artigo conta com mais sete seções. A segunda seção é uma revisão da literatura. A terceira trata do indicador de impacto e das experimentações. A quarta trata da metodologia. A quinta e a sexta apresentam, respectivamente, as covariadas e os resultados estimados. Por fim, a conclusão e sugestões para pesquisas futuras são apresentadas.

\section{Revisão da literatura}

Aries e Newsham (2008) revisaram cerca de 50 trabalhos contendo estimativas da economia de energia atribuída ao horário de verão, além de descrições de externalidades positivas e negativas relacionadas com indicadores de saúde, crime e trânsito ${ }^{8}$. Focando na economia de energia, os valores modais são de que a medida reduziria o consumo agregado em $0,5 \%$. Além disso, os trabalhos revisados invariavelmente apontam que o procedimento melhora a segurança do sistema elétrico interligado ao dispersar a demanda ao longo do dia e entre regiões. Para o caso brasileiro, como pode ser visto em Montalvão (2005) e ONS (2012; 2013), há indicativos alinhados com essas conclusões.

Um fato frisado em Aries e Newsham (2008) e Kellogg e Wolff (2008) é que essas estimativas costumam ser feitas pela comparação da extrapolação do consumo observado antes da mudança do horário com o consumo observado logo depois, o que pode gerar resultados enviesados ${ }^{9}$. Considerando isso, Kellogg e Wolff (2008) propuseram o uso do arcabouço de inferência causal de Neyman-Rubin para estimar consistentemente os efeitos do horário de verão.

Nesse arcabouço, $Y_{i 1}$ denota o resultado de um indicador de impacto (e.g., consumo de energia elétrica) se a unidade $i$ (e.g., um estado) recebe o tratamento (e.g., exposição ao horário diferenciado), $Y_{i 0}$ denota o resultado se a unidade $i$ não recebe o tratamento, e o efeito causal é definido por $Y_{i 1}-Y_{i 0}$. Assim, o problema fundamental de inferência causal é que se observa apenas $Y_{i 1}$ ou $Y_{i 0}$ para cada unidade $i$, sendo o valor observado o factual e outro o contrafactual (Rubin, 2005).

8 Para uma abordagem sobre os efeitos sociais do horário de verão sobre a saúde da população, ver: Harrison (2013); Janszky e Ljing (2008); Barnes e Wagner (2009). Sobre acidentes de transito e criminalidade, ver: Coate e Markowitz (2004); Doleac e Sanders (2015); Franco et al. (2015).

9 Comparações do tipo antes/depois raramente geram medidas de efeito causal - detalhes, por exemplo, em Meyer (1995) ou Sekhon (2008). 
Se existe um número suficientemente grande de unidades elegíveis ao tratamento, sendo que parte delas efetivamente foi tratada (grupo de tratamento) e parte não foi (grupo de controle), uma forma de se inferir um efeito causal médio é estimar a regressão $Y_{i}=\beta_{0}+\beta_{1} T_{i}+\varepsilon_{i}$, onde $Y_{i}$ é o valor observado do indicador de impacto da unidade $i, \beta$ 's são parâmetros, $T_{i}=1$ é uma dummy indicando o pertencimento ao grupo de tratamento e $\varepsilon_{i}$ é o erro. Nesse caso, $\beta_{0}$ representa a média do grupo de controle e $\beta_{1}$ representa o efeito causal médio se, como condição necessária, $T_{i}$ for definido de forma experimental - detalhes em Rubin (2005).

Todavia, no caso em tela $T_{i}$ não costuma ser definido de forma experimental, já que comumente todas as unidades elegíveis são tratadas - i.e., $T_{i}=1$ sempre que a posição geográfica implica em muita iluminação solar no verão. Consequentemente, a consistência da estimativa do efeito causal dependerá de hipóteses de identificação que fundamentam o modelo econométrico. Então, para usar um mínimo de hipóteses, Kellogg e Wolff (2008) propuseram a exploração de situações quase-experimentais que façam com que algumas unidades elegíveis momentaneamente adotem (ou não adotem) o horário de verão.

Uma dessas situações ocorreu no verão de 2000/01 por conta das Olimpíadas de Sydney, quando um dos estados australianos que têm o horário alterado atrasou a mudança em algumas semanas para não atrapalhar os jogos. Explorando isso, Kellogg e Wolff (2008) elaboraram um painel contendo o consumo agregado de energia elétrica por cidades, com observações por hora de cada dia desse verão e dos anterior e posterior. Isso possibilitou o uso de regressões de diferenças-em-diferenças, concluindo que o equivalente do ONS na Austrália superestimava o efeito causal do horário de verão.

Na mesma linha, Kotchen e Grant (2011) exploraram outro quase-experimento ocorrido em 2006 no estado norte-americano de Indiana. Assim, os autores montaram um painel com observações mensais do consumo de energia de milhares de famílias, e estimaram regressões de diferenças-em-diferenças concluindo que o horário de verão não causaria economia nesse caso, mas causaria dispersão da demanda entre regiões.

Explorando uma base de dados com informações do consumo diário de energia elétrica de cerca de duas mil famílias norte-americanas, Sexton e Beatty (2014) aplicaram regressões na descontinuiade dos dias imediatamente anteriores e posteriores ao início e fim do horário de verão. Nesse 
exercício, os autores também não encontraram evidência de que o horário de verão causaria economia, mas encontraram evidência de que causaria dispersão da demanda ao longo do dia.

Na revisão da literatura, Kellogg e Wolff (2008), Kotchen e Grant (2011) e Sexton e Beatty (2014) foram os únicos trabalhos encontrados que estudaram os impactos do horário de verão sobre o consumo de energia usando técnicas decorretes do arcabouço de Neyman-Rubin. Dessa forma, para o caso do Brasil, possivelmente a análise que seguirá aqui é inédita.

\section{Indicador de impacto e quase-experimentos}

Essa pesquisa demandou uma base de dados do consumo de energia elétrica com frequência mensal porque o verão sobrepõe anos distintos no hemisfério sul, e com unidades de observação desagregadas por UFs porque são as unidades de tratamento no Brasil ${ }^{10}$. Além disso, como há cerca de 80 empresas de distribuição de energia elétrica no país, sendo que existem UFs atendidas por mais de uma empresa e empresas que atendem mais de uma UF, a pesquisa também demandou uma padronização na coleta das informações. Dessa forma, aproveitou-se a base de dados compilada desde 2004 pela EPE contendo o consumo agregado mensal de energia elétrica por UF. ${ }^{11}$

Considerando então a estrutura dos dados e a revisão da literatura, estipulou-se que o indicador de impacto ora analisado será o consumo agregado mensal de energia elétrica per capita, ou sua média em determinado verão - i.e., uma medida relacionada à eficiência energética que é passível de medição. Além disso, considerando que o tratamento é aplicado entre o final outubro e o início de fevereiro, os meses de verão aqui considerados serão novembro, dezembro e janeiro - quando não há sobreposição de dias com/sem o horário diferenciado. ${ }^{12}$

10 Se houvesse disponibilidade, a pesquisa poderia usar uma frequência semanal, diária ou horária e com unidades de observação desagregadas por municípios ou famílias como alguns trabalhos descritos em Aries e Newsham (2008).

11 Nessa base não há desagregação com frequência mensal por classe de consumo - ver http://epe.gov.br/mercado.

12 Esse é o indicador mais utilizado pela Agência Internacional de Energia em seus relatórios - ver https://www.iea.org. Aries e Newsham (2008) e Kellogg e Wolff (2008) argumentam que o indicador de impacto ideal para se analisar o horário de verão seria o consumo per capita usado na iluminação, refletindo a razão output/input mais bem relacionada ao objetivo da 
Primeiro se considera uma área aqui chamada de "Brasil meridional", formada pelas UFs que sempre adotam o horário diferenciado. Analogamente, também se considera uma área aqui chamada de "Brasil setentrional", equivalente às regiões Norte e Nordeste, desconsiderando a Bahia e o Tocantins. A Figura 1 ilustra essas regiões, os estados que pertencem à região considerada como limítrofe do horário de verão, bem como apresenta a informação de quantas vezes cada UF experimentou o horário entre os verões de 1988/89 e 2015/16.

Figura 1 Número de vezes em que as UFs tiveram o horário alterado entre os verões de 1988/89 e 2015/16 (no total de 28 verões), considerando as áreas setentrional, meridional e limítrofe*.

UFs setentrionais:

As UFs setentrionais adotaram 0

Acre,

Rondônia,

Amazonas,

Roraima,

Pará,

Amapá,

Maranhão,

Piauí,

Ceará,

Rio Grande do Norte,

Paraíba,

Alagoas,

Sergipe.

UFs meridionais:

Mato Grosso do Sul,

Goiás,

Distrito Federal,

Minas Gerais,

Espírito Santo,

Rio de Janeiro,

São Paulo,

Paraná,

Santa Catarina,

Rio Grande do Sul. horário diferenciado no máximo 4 vezes desde o verão 1988/89.

Fonte: Observatório Nacional. Elaboração própria.

* Horários de verão adotados no limítrofe analisado: Bahia - todos até 2002/03, e depois em 2011/12; Tocantins - em 1989/90, todos entre 1995/96 e 2002/2003, e em 2012/13; Mato Grosso - não adotou apenas em 2003/04.

Complementando as informações apresentadas na Figura 1, a Tabela 1 apresenta, por verão, a média do indicador de impacto por áreas e para

medida, mas raramente se dispõe de tal especificidade. A população considerada no denominador do indicador é a estimada pelo IBGE para o TCU, tendo para o verão de 2004/05 o valor de 2004, e assim sucessivamente. 
os estados da Bahia e do Tocantins. Nela, pode-se notar que a média do consumo per capita no Brasil meridional é quase o dobro da média no Brasil setentrional, o que é consequência do número relativamente maior de equipamentos elétricos em uso - em particular, há relativamente mais consumidores (no comércio, indústria e agricultura) que usam a eletricidade como insumo produtivo. Complementarmente, ao observar que ao longo dos verões o indicador cresceu em todas as regiões analisadas, é possível vislumbrar o aumento generalizado do uso de equipamentos elétricos no país. ${ }^{13}$

Tabela 1 Consumo per capita de energia elétrica ( $\mathrm{kWh} / \mathrm{mês})$ por verão e sua variação (\%) em relação ao verão anterior por áreas selecionadas, de 2004/05 a 2014/2015

\begin{tabular}{|c|c|c|c|c|c|c|c|c|}
\hline \multirow[t]{2}{*}{ Verão } & \multicolumn{4}{|c|}{ Consumo em kWh ao mês* } & \multicolumn{4}{|c|}{ Variação em relação ao verão anterior (\%) } \\
\hline & $\begin{array}{r}*{ }^{*} \text { Brasil } \\
\text { meridi- } \\
\text { onal }\end{array}$ & $\begin{array}{r}* * \text { Brasil } \\
\text { setentri- } \\
\text { onal }\end{array}$ & Bahia & Tocantins & $\begin{array}{r}\text { Brasil } \\
\text { meridi- } \\
\text { onal }\end{array}$ & $\begin{array}{r}\text { Brasil } \\
\text { setentri- } \\
\text { onal }\end{array}$ & Bahia & Tocantins \\
\hline $2004 / 05$ & 188,2 & 98,0 & 110,1 & 58,2 & & & & \\
\hline $2005 / 06$ & 191,2 & 100,9 & 110,6 & 58,5 & 1,6 & 3,0 & 0,5 & 0,5 \\
\hline $2006 / 07$ & 197,8 & 104,6 & 111,1 & 61,6 & 3,5 & 3,7 & 0,5 & 5,3 \\
\hline $2007 / 08$ & 212,2 & 111,3 & 122,9 & 72,5 & 7,3 & 6,4 & 10,6 & 17,7 \\
\hline $2008 / 09$ & 202,0 & 111,0 & 115,1 & 73,6 & $-4,8$ & $-0,3$ & $-6,3$ & 1,5 \\
\hline $2009 / 10$ & 212,0 & 114,5 & 118,7 & 80,9 & 5,0 & 3,2 & 3,1 & 9,9 \\
\hline 2010/11 & 225,9 & 120,1 & 125,6 & 85,6 & 6,6 & 4,9 & 5,8 & 5,8 \\
\hline $2011 / 12$ & 228,3 & 124,4 & $* * * * 124,8$ & 87,3 & 1,1 & 3,6 & $* * * *-0,6$ & 2,0 \\
\hline 2012/13 & 238,1 & 127,3 & 131,1 & $* * * 96,5$ & 4,3 & 2,3 & 5,0 & $* * * * 10,5$ \\
\hline 2013/14 & 238,6 & 130,1 & 131,8 & 101,8 & 0,2 & 2,2 & 0,5 & 5,5 \\
\hline $2014 / 15$ & 238,9 & 129,8 & 141,2 & 110,3 & 0,1 & $-0,2$ & 7,1 & 8,3 \\
\hline
\end{tabular}

Fonte: EPE e IBGE. Elaboração própria.

* Razão entre a soma do consumo agregado nos meses de novembro, dezembro e janeiro e a população do conjunto de UFs, ou estado no caso da Bahia e do Tocantins, dividido por 3.

** Refere-se ao conjunto das UFs das regiões Sul, Sudeste e Centro-Oeste, excluindo-se o Mato Grosso.

*** Refere-se ao conjunto das UFs das regiões Norte e Nordeste, excluindo-se a Bahia e o Tocantins.

**** Indica o verão do quase-experimento.

Como o indicador em tela é uma medida relacionada à eficiência energética, então sua variação apontaria ganho/perda de eficiência. Assim, note13 Detalhes nos Anuários Estatísticos de Energia Elétrica: http://www.epe.gov.br/AnuarioEstatisticodeEnergiaEletrica. 
-se que nos verões dos experimentos o indicador na Bahia cai 0,6\% (em relação ao verão anterior) e no Tocantins sobe $10,5 \%$. Todavia, ressalta-se que o ponto trazido do arcabouço de Neyman-Rubin é que esses percentuais não podem ser lidos como efeito causal do tratamento, pois se estaria desconsiderando uma miríade de elementos contemporâneos que também afetariam a eficiência energética - e.g., maiores rendas refletindo mais uso de equipamentos elétricos ou menores temperaturas refletindo menos uso do ar condicionado - a seção seguinte discute esse ponto.

\section{Metodologia}

A proposta é aplicar duas metodologias de identificação de efeito causal baseadas no arcabouço de Neyman-Rubin: diferenças-em-diferenças, seguindo Kellogg e Wolff (2008) e Kotchen e Grant (2011); e; controle sintético, seguindo Imbens e Wooldridge (2009) e Abadie, Diamond e Hainmueller (2015), que argumentam que esta complementaria a primeira quando há poucas unidades experimentadas, como é o caso aqui.

A ideia das diferenças-em-diferenças é baseada em três passos. Primeiro, toma-se a diferença da esperança condicionada do indicador de impacto nas unidades tratadas entre o verão da experimentação e os demais verões, medindo a variação da eficiência energética sob o tratamento. Segundo, repete-se o procedimento nas unidades de controle, medindo a variação da eficiência energética contemporânea ao tratamento, mas decorrente de outros fatores. Terceiro, toma-se a diferença dessas diferenças, o que isolaria o efeito causal.

Um ponto fundamental nesse sentido é a definição dos grupos de comparação. Assim, como no período observado as UFs do Brasil meridional sempre adotaram o horário diferenciado, e as do Brasil setentrional nunca adotaram, algumas perspectivas são dadas pelo produto cartesiano das seguintes abordagens: o grupo de tratamento pode conter só a Bahia, só o Tocantins ou ambos; e, o de controle pode conter só as UFs meridionais, só as setentrionais ou todas fora do limítrofe.

Considerando apenas uma unidade no tratamento, a operacionalização da metodologia passa pelas seguintes definições: $Y_{i t}$ é a média do consumo agregado mensal de energia elétrica per capita da UF $i$ no verão $t ; T_{i}$ é uma dummy se $i=i^{*}$, onde $i^{*}$ é a UF experimentada; $V_{t}$ é uma dummy se $t=t^{*}$, onde 
$t^{*}$ é o verão do experimento; $X_{i t}$ é um vetor de covariadas; e, $E(\cdot)$ indica a expectância. Assim, as diferenças-em-diferenças serão:

$$
\begin{aligned}
& D D=\left[E\left(Y_{i t} \mid T_{i}=1, V_{t}=1, X_{i t}\right)-E\left(Y_{i t} \mid T_{i}=1, V_{t}=0, X_{i t}\right)\right]- \\
& -\left[E\left(Y_{i t} \mid T_{i}=0, V_{t}=1, X_{i t}\right)-E\left(Y_{i t} \mid T_{i}=0, V_{t}=0, X_{i t}\right)\right]
\end{aligned}
$$

Explicitando-se as dummies, cada elemento de expectância em (1) pode ser escrito como $E\left(Y_{i t} \mid X_{i t}\right)=\beta_{0}+\beta_{1} T_{i}+\beta_{2} V_{t}+\beta_{3} T_{i} V_{t}$, de forma que ocorrerá $D D=\beta_{3}$. Além disso, explorando os dados em painel é possível controlar os efeitos fixos das unidades de controle, $f_{i \neq i}$, e dos verões sem experimentação, $v_{t \neq i}$, de forma a se trabalhar com a seguinte regressão linear:

$$
Y_{i t}=\beta_{0}+\beta_{1} T_{i}+\beta_{2} V_{t}+\beta_{3} T_{i} V_{t}+\sum_{k \geq 4} \beta_{k} x_{k i t}+f_{i \neq i}+v_{t \neq t^{*}}+\varepsilon_{i t}
$$

em que: $x_{k i t}, f_{i \neq i^{*}}, v_{t \neq t^{*}} \in X_{i t} ; \varepsilon_{i t}$ é um termo de erro; e, os demais elementos seguem as definições anteriores. Assim, o valor estimado para $\beta_{3}$ na equação (2) indicaria o ganho/perda de eficiência energética causada pela adoção do horário de verão (na Bahia ou no Tocantins) em relação aos controles.

Complementarmente, considerando concomitantemente a Bahia e o Tocantins no grupo de tratamento é possível se reformular a equação (2) da seguinte forma:

$$
Y_{i t}=\beta_{3} T_{i=B A} V_{t=2011 / 12}+\tilde{\beta}_{3} \tilde{T}_{i=T O} \tilde{V}_{t=2012 / 13}+\sum_{k \geq 4} \beta_{k} x_{k i t}+\tilde{f}_{i}+\tilde{v}_{t}+\varepsilon_{i t}
$$

em que: $T_{i=B A}$ e $\tilde{T}_{i=T O}$ são dummies indicando que a unidade de observação é a Bahia ou o Tocantins, respectivamente; $V_{t=2011 / 12}$ e $\tilde{V}_{t=2012 / 13}$ são dummies indicando os verões dos experimentos; $\tilde{f}_{i}$ e $\tilde{v}_{t}$ são efeitos fixos para UFs e verões; e, os demais elementos seguem as definições anteriores. Assim, os valores estimados para $\beta_{3}$ e $\tilde{\beta}_{3}$ na equação (3) indicariam o ganho/perda de eficiência energética causada pela adoção do horário de verão na Bahia e no Tocantins, respectivamente, em relação aos controles.

Nota-se que ao invés da definição impositiva das UFs de controle, uma definição por pareamento seria mais adequada para a interpretação de $\beta_{3}$ e $\tilde{\beta}_{3}$ como efeito causal - detalhes, por exemplo, em Khandker, Koolwal e Samad (2010). Todavia, como há uma única unidade tratada 
em cada verão, o pareamento selecionaria poucos controles, implicando na perda de muitos graus de liberdade. Para evitar esse custo em benefício da checagem dos resultados, uma alternativa é sintetização das unidades tratadas. ${ }^{14}$

Inicialmente proposta por Abadie e Gardeazabal (2003), a metodologia de sintetização começa com a definição dos valores do indicador de impacto com e sem a intervenção, $Y_{i t}^{(1)}$ e $Y_{i t}^{(0)}$, respectivamente. Considera-se então que nenhuma unidade analisada é tradada no período pré-intervenção, logo $Y_{i t}^{(1)}=Y_{i t}^{(0)}$ para todo $i$ e $t<t^{*}$, e consequentemente, definindo uma dummy $I_{i t}=1$ se $i=i^{*}$ e $t \geq t^{*}$, o valor observado do indicador de impacto é $Y_{i t}=Y_{i t}^{(0)}+\alpha_{t} I_{i t}$. Com essa estrutura, $\alpha_{t}$ seria o efeito causal da intervenção.

Para estimar $\alpha_{t}$, Abadie, Diamond e Hainmueller (2010) consideram $Y_{i t}=\theta_{t} Z_{i}+\xi_{i t}$, em que $\theta_{t}$ e $Z_{i}$ são vetores linha de parâmetros e coluna de covariadas, respectivamente, e $\xi_{i t}$ é um termo que agrega fatores temporais, erros e omissões. Dessa forma, os autores demonstram que se: (a) há um número suficientemente grande de períodos pré-intervenção observados; e, (b) existem pesos $w_{i} \geq 0$ para todo $i \neq i^{*}$, tais que $\sum_{i \neq i^{*}} w_{i}=1$, $\sum_{i \neq *^{*} W_{i}} Y_{i t}=Y_{i^{*} t}$ e $\sum_{i \neq i^{*}} W_{i} Z_{i t}=Z_{i^{*} t}$ e para todo $t<t^{*}$; então um estimador de efeito causal é $\hat{\alpha}_{t}=Y_{i^{*} t}-\sum_{i \neq i^{*}} W_{i} Y_{i t}$, em que $\sum_{i \neq *^{*}} w_{i} Y_{i t}$ é o sintético de $Y_{i^{*} t^{*}}$. Assim, toda unidade $i$ seria adequadamente selecionada para o grupo de controle se $w_{i} \neq 0$.

Os exercícios de Abadie, Diamond e Hainmueller (2010, 2015) sugerem que é necessário observar ao menos 20 períodos para se operacionalizar a metodologia satisfazendo a condição (a). No caso ora analisado, já que há três meses sem sobreposição de dias com/sem o horário de verão, com os dados da EPE é possível observar 21 e 24 períodos pré-intervenção nos casos da Bahia e do Tocantins, respectivamente, se o controle for do Brasil setentrional e a unidade temporal for o mês ${ }^{15}$. Complementarmente, para determinar o sintético esses autores propõem o critério da minimização da soma dos quadrados ponderados dos desvios das condições (b), da seguinte forma:

14 Selecionar um grupo de controle adequado evitando a perda de graus de liberdade é uma motivação comum para o controle sintético em detrimento as diferenças-em-diferenças com pareamento quando há poucas unidades tratadas - detalhes em Imbens e Wooldridge (2009) ou Abadie, Diamond e Hainmueller (2015).

15 As UFs do Brasil meridional não podem integrar o sintético sem ferir as hipóteses do modelo, porque sempre adotaram o horário de verão no período observado. 
Min. (para todo $w_{i}$ tal que $i \neq i^{*}$ e todo $q_{k}$ tal que $\left.k=0, \ldots, K\right)$ s.r

$$
\begin{aligned}
& q_{0}\left(\bar{Y}_{i}-\sum_{i \neq i} w_{i} \bar{Y}_{i}\right)^{2}+\sum_{k=1}^{K} q_{k}\left(z_{k i}-\sum_{i \neq i} w_{i} z_{k i}\right)^{2} \\
& \sum_{i \neq i} w_{i}=1 ; \sum_{k=0}^{K} q_{k}=1
\end{aligned}
$$

em que: $q_{k}$ são as ponderações determinadas dentro do procedimento de minimização; $\bar{Y}_{i}$ é a média do indicador de impacto nos períodos pré-intervenção; $K$ é o número de covariadas consideradas; e, $z_{k i} \in Z_{i}$, podendo ser médias das covariadas.

Para se fazer a inferência de $\hat{\alpha}_{t}$ decorrente dos valores de $w_{i}$ encontrados, Abadie, Diamond e Hainmueller (2010, 2015) sugerem a comparação de resultados placebos - i.e., retira-se sequencialmente toda unidade $i$ tal que $w_{i} \neq 0$ econtrando novas soluções para (4) - se as soluções forem semelhantes, isso indicaria a consistência do contrafactual estimado.

Dessa forma, adiante se apresentará as estimativas do efeito causal do horário de verão através das diferenças-em-diferenças - $\beta_{3}$ e $\tilde{\beta}_{3}$ pelas equações (2) e (3) - e do controle sintético - $\hat{\alpha}_{t}$ decorrente da resolução do problema (4). Mas antes é necessária uma discussão das covariadas a serem utilizadas nessas estimações, o que é feito na sequência.

\section{Covariadas}

Seguindo Kellogg e Wolff (2008) e Kotchen e Grant (2011), o foco da análise é a estimação do contrafactual do consumo equilíbrio de energia elétrica. Portanto, o foco está na forma reduzida de um modelo estrutural de oferta e demanda de energia, e a inclusão de preços nas equações (2) e (3) é inoportuna por conta da endogeneidade. Considerando isso, o vetor de covariadas deve conter um indicador de renda ou produção e outros deslocadores do equilíbrio de mercado.

A Tabela 2 apresenta as covariadas tabuladas e seus valores de referência no verão de 2013/14 - que é o mais recente que contém todas as informações. Na primeira linha repete-se o consumo per capita ( $\mathrm{kWh}$ ) de energia elétrica mensal exposto anteriormente, atentando que o valor do Brasil meridional é cerca do dobro do Brasil setentrional, Bahia e Tocantins, e notando que o PIB per capita em 2013 (R\$ mil) segue essa proporciona- 
lidade. Além disso, em IBGE (2015) pode ser verificado que isso é regular e na literatura há uma boa documentação da correlação entre essas duas variáveis, portanto o PIB deve ser considerado na estimação dos contrafactuais em análise. ${ }^{16}$

Outro deslocador do equilíbrio seria o perfil dos consumidores. Pois enquanto o consumo residencial seria elástico ao horário de verão, parte do consumo no comércio, indústria e agricultura seria inelástico porque é fator de produção - detalhes em Aries e Newsham (2008). Logo, deve ser feito um esforço para se controlar esses perfis - lembrando que na base de dados não é possível desagregar o consumo das classes por mês para analisar os verões.

Nesse sentido, usando os Anuários Estatísticos de Energia Elétrica da EPE é possível tabular o número de consumidores por UF em dezembro i.e., medidores de consumo faturados pelas distribuidoras. Assim, note-se na Tabela 2 que os consumidores residenciais representam mais de $80 \%$ do total em todas as áreas consideradas, seguidos das classes comerciais e rurais. Nos Anuários também é possível notar que essas proporções são regulares entre os anos e UFs, exceto para o Tocantins, que possui uma tendência de troca de medidores de consumo faturados na classe rural para a residencial. Considerando isso, como controles nas equações (2) e (3) podem ser usadas essas proporções sob a hipótese de que o número de medidores em um dado verão é suficientemente próximo do observado em dezembro.

Outros deslocadores do equilíbrio explorados na literatura são a densidade populacional (hab./ $/ \mathrm{Km}^{2}$ ) em 2013, o tempo médio diário de iluminação solar (horas) no verão de 2013/14 e a média da temperatura diária $\left({ }^{\circ} \mathrm{C}\right)$ no verão de 2013/14. Sendo esperado que os dois primeiros sejam negativamente correlacionados com o consumo, já que em ambos os casos mais pessoas fariam uso de um mesmo espaço iluminado, enquanto maiores temperaturas estariam positivamente correlacionadas com o consumo de energia elétrica por conta do uso dos equipamentos de climatização.

A densidade populacional não é uma boa covariada nas equações (2) e (3), porque o denominador é fixo e o numerador apresenta uma tendência ser vistos, por exemplo, em Schmidt e Lima (2004), Mattos e Lima (2005), Irffi et al. (2009) ou Neto, Corrêa e Perobelli (2016). 
linear (i.e., a população cresce regularmente), o que acarreta em multicolineariade com os efeitos fixos dos verões. Entretanto, ela é útil na sintetização das trajetórias de consumo no problema (4).

Já as variáveis climáticas podem ser tabuladas para as principais cidades com os dados do Instituto Nacional de Meteorologia (INMET), que dispobibiliza registros diários de várias estações meteorológicas. Assim, na Tabela 2 note-se que a média diária de horas de sol no verão em Porto Alegre é de 13,7, enquanto em Boa Vista é de 11,9 - uma diferença de 108 minutos enquanto os valores em Salvador e Palmas são intermediários a estes. Como essas diferenças são precisamente o motivador do horário de verão, é interessante controlá-las. Entretanto, o tamanho do dia e as temperaturas também não são boas covariadas nas equações (2) e (3) porque suas variâncias são pequenas entre verões em uma mesma UF, causando multicolinearidade com os efeitos fixos. De toda forma, estas são covariadas úteis no problema (4).

Tabela 2 Covariadas utilizadas e valores de referência no verão de 2013/14 por áreas selecionadas

\begin{tabular}{l|r|r|r|r}
\hline Covariadas & $\begin{array}{r}\text { Brasil } \\
\text { meridi- } \\
\text { onal }\end{array}$ & $\begin{array}{r}\text { Brasil } \\
\text { setentri- } \\
\text { onal }\end{array}$ & Bahia & Tocantins \\
\hline Consumo per capita ao mês (kWh) & 238,6 & 130,1 & 131,8 & 101,8 \\
\hline PIB per capita em 2013 (R\$ mil) & 33,7 & 14,0 & 13,6 & 16,1 \\
\hline Classes de consumidores em 2013 (\%): & & & & \\
\hline - Residenciais & 82,9 & 84,3 & 87,8 & 88,2 \\
\hline - Comerciais & 8,4 & 7,1 & 7,0 & 6,2 \\
\hline - Rurais & 6,8 & 6,9 & 3,7 & 4,0 \\
\hline - Industriais & 1,1 & 0,3 & 0,4 & 0,4 \\
\hline - Outros & 0,8 & 1,4 & 1,1 & 1,2 \\
\hline Densidade populacional em 2013 (hab./Km2) & 56,7 & 12,3 & 26,6 & 5,4 \\
\hline Média de horas de sol ao dia no verão de 2013/14 & $* 13,7$ & ${ }^{*} 11,9$ & ${ }^{*} 12,7$ & $* 12,5$ \\
\hline Média das temperaturas diárias no verão de 2013/14 ( $\left.{ }^{\circ} \mathrm{C}\right):$ & & & & \\
\hline - Mínima & $* 19,9$ & $* * 24,6$ & $* * * 22,9$ & $* * * * 22,1$ \\
\hline - Máxima & $* 31,0$ & $* * 34,5$ & ${ }^{* * *} 30,9$ & $* * * * 32,0$ \\
\hline
\end{tabular}

Fonte: EPE (2015), IBGE (2015), INMET(2016). Elaboração própria.

*Porto Alegre; ** Boa Vista; *** Salvador; **** Palmas. 


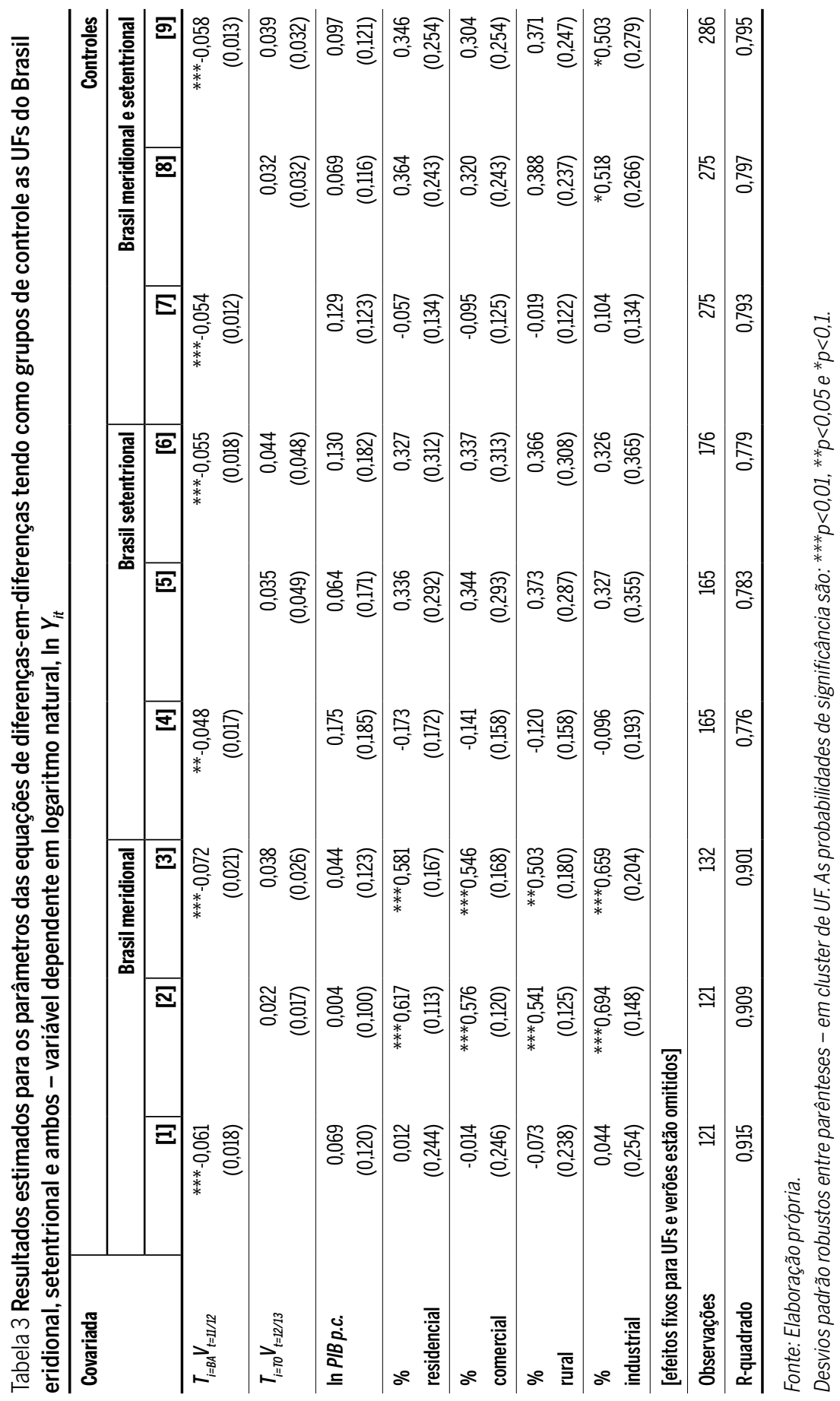




\section{Resultados estimados}

A Tabela 3 apresenta os resultados estimados para as equações de diferenças-em-diferenças. Assim como em Kellogg e Wolff (2008) e Kotchen e Grant (2011), para que os parâmetros sejam interpretados em termos de elasticidade (ou semi-elasticiade) as especificações usam a variável dependente em logaritimo e as explicativas em logaritimo, dummy ou entre 0 e 100 no caso de percentual. Além disso, como discutido em Cameron e Miller (2015), para mitigar as autocorrelações residuais a inferência considera variâncias estimadas em cluster de UF.

As colunas [1], [2] e [3] apresentam os resultados considerando no grupo de tratamento só a Bahia, só o Tocantins e ambos, respectivamente, tendo como controles as UFs do Brasil meridional. Essas estimativas indicam que a adoção do horário de verão teria causado algo como $6,1 \mathrm{kWh}$ ou $7,2 \%$ de redução do consumo agregado de energia elétrica per capita na Bahia, e indicam ausência de impacto estatisticamente significante no Tocantins.

Já as colunas [4], [5] e [6] apresentam os resultados tendo no controle as UFs do Brasil setentrional, e as colunas [7], [8] e [9] consideram no controle todas as UFs fora do limítrofe. Nessas regressões os resultados indicam que a adoção do horário teria causado cerca de $5 \%$ de redução do consumo per capita na Bahia, e não teria causado impacto no Tocantins.

As estimativas dos parâmetros do PIB per capita não se mostraram estatisticamente significantes em nenhuma das especificações ${ }^{17}$. E as estimativas dos parâmetros dos perfis de consumidores se mostraram estatisticamente significantes em algumas regressões onde o Tocantins figurou no grupo de tratamento - tendo em vista que este estado vem apresentando mudanças desses perfis no período analisado.

Como argumentado antes, para checar esses indicativos de efeito causal usa-se o controle sintético com todas as covariadas tabuladas. Assim, a Tabela 4 apresenta os pesos $\left(w_{i}\right)$ que solucionam o problema (4), sendo que para as UFs não listadas encontrou-se $w_{i}=0$. Note-se que os estados que sintetizaram a Bahia foram Alagoas, Pará e Pernambuco, e os que sintetizaram o Tocantins foram Acre, Piauí, Rondônia e Sergipe.

17 O PIB per capita está a preços constantes de 2013 considerando o deflator implícito, e para 2014 o valor de 2013 foi repetido porque o IBGE ainda não havia divulgado o número. Para o verão de 2004/05 usa-se o PIB de 2004, e assim sucessivamente. 
Tabela 4 Pesos estimados $\left(\boldsymbol{w}_{i}\right)$ através do controle sintético para o grupo de controle estados setentrionais*

\begin{tabular}{l|r|r|r|r|r|r|r}
\hline \multirow{2}{*}{$\begin{array}{l}\text { Unidades } \\
\text { tratadas }\end{array}$} & \multicolumn{10}{c}{ Unidades de controle } \\
\cline { 2 - 9 } & AC & AL & PA & PE & PI & RO & SE \\
\hline BA & 0,272 & 0,241 & 0,487 & & & \\
\hline T0 & 0,425 & & 0,151 & 0,034 \\
\hline
\end{tabular}

Fonte: Elaboração própria.

* Para UFs não listadas encontrou-se $\mathrm{w}_{\mathrm{i}}=0$ na resolução do problema (4).

Como em Abadie, Diamond e Hainmueller (2010, 2015), para checar o quão bem o controle sintético mimetiza as unidades tratadas, a Tabela 5 apresenta a média do indicador de impacto entre todos os meses dos verões observados, $\bar{Y}_{i}$, e a média sintetizada pelos pesos, $\sum_{i \neq i} w_{i} \bar{Y}_{i}$, e da mesma forma faz-se para as covariadas. Notando que todos os indicadores parecem ter sido bem mimetizados.

Tabela 5 Médias observadas $\left(\bar{Y}_{i^{*}}\right.$ e $\left.z_{k^{*}}\right)$ e $\operatorname{sintetizadas}\left(\sum_{i=i^{*}} w_{i} \bar{Y}_{i}\right.$ e $\left.\sum_{i=i^{*}} w_{i} z_{k i}\right)$ para os períodos observados pré-intervenção nos estados da Bahia e do Tocantins

\begin{tabular}{l|r|r|r|r}
\hline Indicador de impacto e covariadas & & Bahia & & Tocantins \\
\cline { 2 - 5 } & Observado & Sintético & Observado & Sintético \\
\hline Consumo de energia per capita (kWh) & 116,30 & 116,27 & 72,26 & 72,33 \\
\hline PIB per capita (R\$ mil de 2013) & 12,26 & 10,80 & 13,80 & 11,59 \\
\hline Consumidores residenciais (\%) & 87,88 & 87,21 & 77,62 & 81,04 \\
\hline Consumidores comerciais (\%) & 6,26 & 6,55 & 7,19 & 7,52 \\
\hline Consumidores rurais (\%) & 3,99 & 4,90 & 12,81 & 9,53 \\
\hline Consumidores industriais (\%) & 0,44 & 0,35 & 0,52 & 0,40 \\
\hline Outras classes de consumidores (\%) & 1,44 & 1,00 & 1,86 & 1,52 \\
\hline Densidade populacional (habitantes/Km ${ }^{2}$ ) & 24,83 & 25,60 & 4,98 & 10,93 \\
\hline Temperatura máxima diária ( $\left.{ }^{\circ} \mathrm{C}\right)$ & 30,92 & 31,21 & 32,41 & 32,86 \\
\hline Temperatura mínima diária ( $\left.{ }^{\circ} \mathrm{C}\right)$ & 22,94 & 22,79 & 22,57 & 22,80 \\
\hline Quantidade de horas de sol por dia & 12,76 & 12,40 & 12,58 & 12,47 \\
\hline
\end{tabular}

Fonte dos dados: EPE (2015), IBGE (2016), INMET(2016) e elaboração própria.

Já a Figura 2 apresenta as trajetórias do consumo de energia per capita na Bahia e no Tocantins nos meses dos verões observados, em que as linhas negras são os factuais e as tracejadas em cinza são contrafactuais sintetizados e placebos nas resoluções do problema (4). Por inspeção visual, o con- 
trole sintético corrobora com os resultados de diferenças-em-diferenças, em que o consumo contrafactual da Bahia teria sido maior que o factual no verão de 2011/12, e que não houve efeito causal no Tocantins no verão de 2012/13.

Segundo ONS (2012) a inclusão da Bahia no horário de verão em 2011/12 teria causado uma economia de $35 \mathrm{GWh}$ ao país, além de ter dispersado picos de demanda regionais e reduzido o risco de "apagões" e o uso de água nos reservatórios hidroelétricos. Porém, pelos resultados da Tabela 3 , o impacto seria de pelo menos de $4,8 \%$ no consumo médio mensal per capita, uma diferença de $6 \mathrm{kWh}$ entre o contrafactual e seu factual - vide Tabela 1 e/ou Figura 2(a). Considerando os três meses de verão aqui analisados e a população de cerca de 14 milhões de pessoas na época, as estimativas provenientes do arcabouço de Neyman-Rubin apontam que a inclusão da Bahia teria causado uma economia mais próxima de 252 GWh. $^{18}$

Complementarmente, segundo ONS (2013) o horário diferenciado teria causado uma economia de $3 \mathrm{GWh}$ ao país em decorrência da inclusão da Tocantins no verão de 2012/13. Todavia, pelas estimativas reportadas aqui não houve impacto estatisticamente significante ${ }^{19}$. Dessa forma, através do arcabouço de inferência causal de Neyman-Rubin essa pesquisa encontrou estimativas alinhadas com as do ONS. Todavia, encontrou-se indicativo de que o ONS pode estar subestimando os impactos da política no caso Bahia.

Levando em conta os motivos ventilados na mídia acerca da participação desses estados na política nos últimos verões, quais sejam, de que a Bahia não participa em decorrência de uma decisão política para agradar a opinião pública, e de que o Tocantins participou apenas para ajustar o horário com a metade sul do país, conclui-se que há indicativos de que o primeiro estado deveria adotar o horário diferenciado e o segundo não. Pois a inclusão da Bahia poderia gerar maior segurança ao sistema nacional de geração e distribuição de energia elétrica, como indicam as notas técnicas do ONS. Já a inclusão do Tocantins não geraria impacto significativo nesse sentido.

18 Resultado do produto entre $6 \mathrm{kWh}$ por três meses por 14 milhões de pessoas.

19 Segundo ONS (2012, 2013), considerando todas as UFs o horário de verão em 2011/12 e 2012/13 teria causado uma economia de 815 e 687 GWh ao país, respectivamente. 
Figura 2 Trajetórias do consumo de energia per capita (kWh) na Bahia e no Tocantins nos meses dos verões observados: as linhas negras são os factuais e as tracejadas em cinza são contrafactuais sintetizados e placebos nas resoluções do problema (4).

\section{a Bahia}

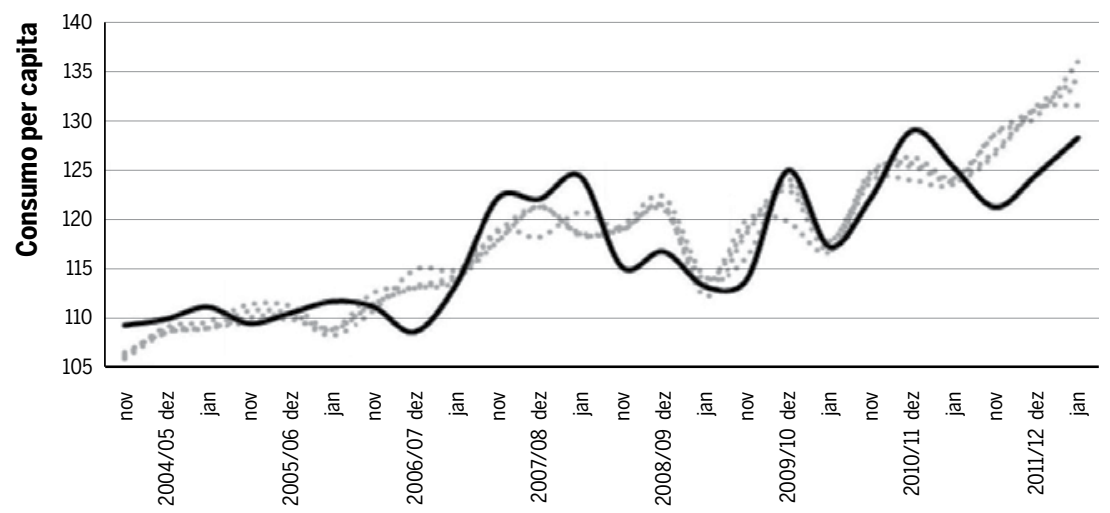

b Tocantins

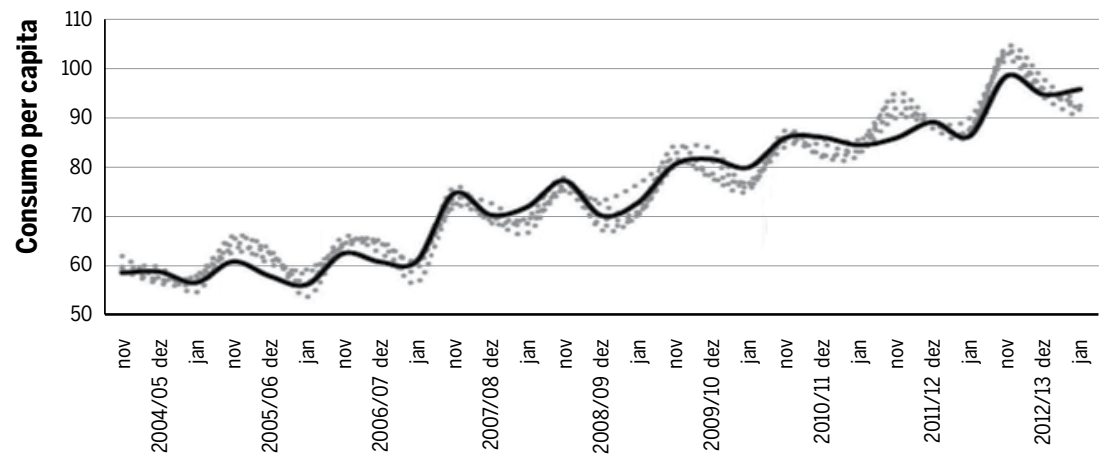

Fonte: Elaboração própria.

\section{Conclusão}

Com o objetivo de contribuir para o debate sobre o limítrofe de adoção do horário de verão no Brasil e tirando proveito dos quase-experimentos que ocorreram nos casos da Bahia e do Tocantins, conclui-se que a política tem efeitos significativos na redução do consumo de energia elétrica no 
primeiro estado, mas no segundo não. Resultado em linha com as notas técnicas do ONS.

Ao se obter um ganho de eficiência energética com a adoção do horário de verão na Bahia, pode-se dar maior segurança ao sistema interligado nacional, poupando investimentos na geração e transmissão de energia elétrica e reduzindo a necessidade de energia proveniente de termoelétricas, mais cara e poluente quando comparado à hidro e eólica. E, ao passo que não há redução significativa no consumo de energia elétrica no Tocantins, para não afetar a rotina das pessoas, manter o horário inalterado pode ser a melhor opção.

Está ainda encoberta a questão do efeito do horário de verão no estado do Mato Grosso. Nesse caso, considerando que parte dos municípios do estado não vem adotando a política nos últimos verões, e adotaram no passado, uma pesquisa futura pode fazer proveito disso para aplicar modelos de diferenças-em-diferenças - semelhante ao que foi feito em Kellogg e Wolff (2008) e Kotchen e Grant (2011) - ou de regressões na descontinuidade - semelhante ao que foi feito em Sexton e Beatty (2014). Isso contribuiria para dar fecho a parte das polêmicas que envolvem o horário de verão no Brasil.

\section{Referências}

ABADIE, A.; DiAMOND, A.; HAINMUELLER, J. (2010). Synthetic Control Methods for Comparative Case Studies: Estimating the Effect of California's Tobacco Control Program. Journal of the American Statistical Association, v. 105, n. 490, p. 493-505.

ABADIE, A.; DIAMOND, A.; HAINMUELLER, J. (2015). Comparative Politics and the Synthetic Control Method. American Journal of Political Science, v. 59, n. 2, p. 495-510.

ABADIE, A.; GARDEAZABAL, J. (2003). The Economic Costs of Conflict: A Case Study of the Basque Country. The American Economic Review, v. 93, n. 1, p. 113-132.

ARIES, M. B.; NEWSHAM, G. R. (2008). Effect of daylight saving time on lighting energy use: A literature review. Energy Policy, v. 36, p. 1858-1866.

BARNES, C. M.; WAGNER, D. T. (2009). Changing to daylight saving time cuts into sleep and increases workplace injuries. Journal of applied psychology, v. 94, n. 5, p. 1305-1317.

CAMERON, A. C.; MILLER, D. L. (2015). A Practitioner's Guide to Cluster-Robust Inference. Journal of Human Resources, v. 50, n. 2, p. 317-372.

COATE, D.; MARKOWITZ, S. (2004). The effects of daylight and daylight saving time on US pedestrian fatalities and motor vehicle occupant fatalities. Accident Analysis and Prevention, v. 36, p. 351-357. 
DOLEAC, J. L.; SANDERS, N. J. (2015). Under the cover of darkness: how ambient light influences criminal activity. The Review of Economics and Statistics, v. 97, p. 1093-1103.

EPE - Empresa de Pesquisa Energética. Anuário Estatístico de Energia Elétrica: 2014. Rio de Janeiro, 2015.

FRANCO, C.; SAMPAIO, G. R.; SAMPAIO, B.; MACHADO, W. T. (2015). Avançar o relógio em uma hora pode evitar acidentes? Evidências da política do horário de verão no Brasil. In: XX Encontro Regional de Economia. Anais... ANPEC e Banco do Nordeste do Brasil, Fortaleza, 2015.

IBGE. (2015). Contas Regionais do Brasil 2010-2013. Contas Nacionais, Número 47.

HARRISON, Y. (2013). The impact of daylight saving time on sleep and related behaviors. Sleep Medicine Reviews, v. 17, n. 4, p. 285-292.

IMBENS, G. W.; WOOLDRIDGE, J. M. (2009). Recent Developments in the Econometrics of Program Evaluation. Journal of Economie Literature, v. 47, n. 1, p. 5-86.

INMET - Instituto Nacional de Meteorologia. Monitoramento Climático Nacional. Brasília, 2016.

IRFFI, G.; CASTELAR, I.; SIQUEIRA, M. L.; LINHARES, F. C. (2009). Previsão da demanda por energia elétrica para classes de consumo na região Nordeste, usando OLS dinâmico e mudança de regime. Economia Aplicada, v. 13, n. 1, p. 69-98.

JANSZKY, I.; LJUNG, R. (2008). Shifts to and from Daylight Saving Time and Incidence of Myocardial Infarction. New England Journal of Medicine, v. 359, p. 1966-1968.

KELLOGG, R.; WOLFF, H. (2008). Daylight time and energy: Evidence from an Australian experiment. Journal of Environmental Economics and Management, v. 56, p. 207-220.

KHANDKER, S. R.; KOOLWAL, G. B.; SAMAD, H. A. (2010). Handbook on Impact Evaluation: Quantitative Methods and Practices. Washington DC: The International Bank for Reconstruction and Development (The World Bank).

KOTCHEN, M. J.; GRANT, L. E. (2011). Does daylight saving time save energy? Evidence from a natural experiment in Indiana. The Review of Economics and Statistics, v. 93, n. 4, p. 1172-1185.

MATTOS, L. B.; LIMA, J. E. (2005). Demanda residencial de energia elétrica em Minas Gerais: 1970-2002. Nova Economia, v. 15, n. 3, p. 31-52.

MEYER, B. D. (1995). Natural and quasi-experiments in economics. Journal of Business \& Economic Statistics, v. 13, n. 2, p. 151-161.

MONTALVÃO, E. (2005). O setor elétrico e o horário de verão. Senado Federal, Consultoria Legislativa, Texto para discussão n. 19.

NETO, A. B.; CORRÊA, W. L.; PEROBELLI, F. S. (2016). Consumo de Energia e Crescimento Econômico: uma Análise do Brasil no período 1970-2009. Análise Econômica, v. 34, n. 65, p. 181-204.

ONS (2012). Término do Horário de Verão 2011/2012. Operador Nacional do Sistema Elétrico, Nota técnica 42/2012.

ONS (2013). Término do Horário de Verão 2012/2013. Operador Nacional do Sistema Elétrico, Nota técnica 22/2013.

RUBIN, D. (2005). Causal Inference Using Potential Outcomes: Design, Modeling, Decisions. 
Journal of the American Statistical Association, v. 100, n. 469, p. 322-331.

SCHMIDT, C. A.; LIMA, M. A. (2004). A Demanda por Energia Elétrica no Brasil. Revista Brasileira de Economia, v. 58, n. 1, p. 67-98.

SEKHON, J. (2008). The Neyman-Rubin Model of Causal Inference and Estimation Via Matching Methods. The Oxford Handbook of Political Methodology, v. 2, p. 1-37.

SEXTON, A. L.; BEATTY, T. K. (2014). Behavioral responses to Daylight Savings Time. Journal of Economic Behavior \& Organization, v. 107, p. 290-307.

\section{Sobre os autores}

FrancisPetterini-f.petterini@ufsc.br

Universidade Federal de Santa Catarina, Florianópolis, Santa Catarina, Brasil. ORCID: https://orcid.org/0000-0003-4410-0970.

DiogoSignor-diogo.signor@ufsc.br

Universidade Federal de Santa Catarina, Florianópolis, Santa Catarina, Brasil. ORCID: https://orcid.org/0000-0003-0979-2342.

PedroSantos - pedro.vmo@gmail.com

Universidade Federal de Santa Catarina, Florianópolis, Santa Catarina, Brasil. ORCID: https://orcid.org/0000-0001-7737-4121.

\section{Sobre o artigo}

Recebido em 25 de maio de 2016. Aprovado em 03 de março de 2017. 